\title{
Spectral Minutiae Representations for Fingerprint Recognition
}

\author{
Haiyun Xu and Raymond N.J. Veldhuis \\ Department of Electrical Engineering, University of Twente \\ PO Box 217, 7500AE Enschede, The Netherlands \\ $\{$ h.xu,r.n.j.veldhuis\}@ewi.utwente.nl
}

\begin{abstract}
The spectral minutiae representation introduced in this paper is a novel method to represent a minutiae set as a fixed-length feature vector, which is invariant to translation, and in which rotation becomes translation that can be easily compensated for. These characteristics enable the combination of fingerprint recognition systems with a template protection scheme that requires a fixed-length feature vector as input. In this paper, we will first introduce the spectral minutiae representation scheme. Then we will present several biometric fusion approaches to improve the biometric system performance by combining multiple sources of biometric information. The algorithms are evaluated on the FVC2000-DB2 database and showed promising results.
\end{abstract}

Keywords-Biometrics, fingerprints, template protection.

\section{INTRODUCTION}

Recognition of persons by means of biometric characteristics is gaining importance due to the high security and user convenience. Among various biometric identifiers, such as face, signature and voice, fingerprint has one of the highest levels of distinctiveness and performance [1] and it is the most commonly used biometric modality. Most fingerprint recognition systems are based on the use of a minutiae set. However, the low comparison (or matching) speed is limiting its application to search large databases. At the same time, the increasing privacy concerns make minutiae template protection a crucial task. The spectral minutiae representation is a method to represent a minutiae set as a fixed-length feature vector, which is invariant to translation, and in which rotation becomes translation that can be easily compensated for [2], [3]. These characteristics enable the combination of fingerprint recognition systems with template protection schemes and allow for a fast minutiae-based matching as well.

In order to apply the spectral minutiae representation with a template protection scheme based on fuzzy commitment and helper data schemes, such as [4] and [5], we also need to quantize the real-valued spectral minutiae features into binary strings. We illustrate a helper data scheme in Figure 1. The spectral minutiae representation scheme is shown as modules 'Feature Extraction' and 'Quantization' in this figure. In module 'Feature Extraction', a fingerprint minutiae set will be transformed into a fixed-length real-valued feature vector using the complex spectral minutiae representation algorithm. In module 'Quantization', the real-valued feature

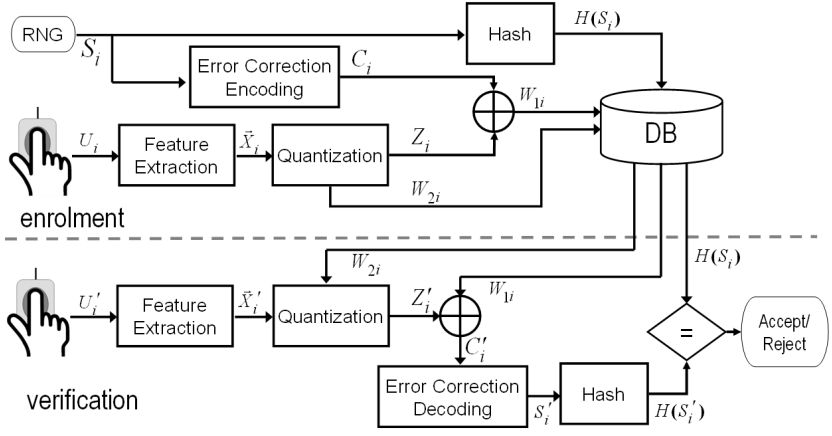

Figure 1. The structure diagram of the helper data scheme.

vector will be quantized into a binary string. A fixed-length binary representation also has additional advantages such as small template storage and high matching speed.

In this paper, we will first introduce the spectral minutiae representation scheme in Section II. Next, in Section III, we will discuss several methods to implement multi-sample fusions to improve the recognition performance of the spectral minutiae representation scheme. Then, we will show the experimental results in Section IV. Finally, we will draw conclusions in Section V.

\section{Spectral Minutiae Representation}

\section{A. Overview}

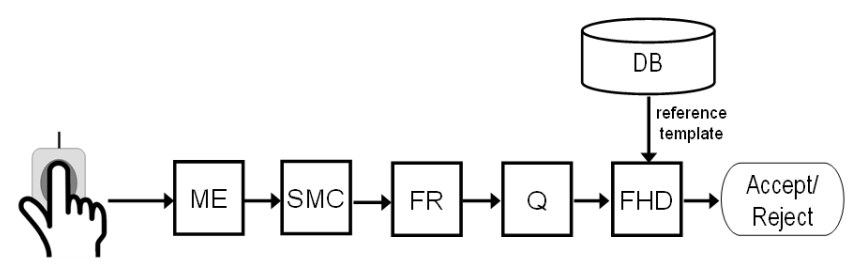

Figure 2. The various processing modules of the spectral minutiae representation scheme. The acronym of each processing modules stands for: ME: Minutiae Extractor. SMC: Complex Spectral Minutiae representation. FR: Feature Reduction. Q: Quantization. FHD: Fractional Hamming Distance.

The spectral minutiae representation scheme introduced in this paper represents a minutiae set into a fixed-length binary string. This enables the combination of a minutiae-based fingerprint recognition system and a template protection 


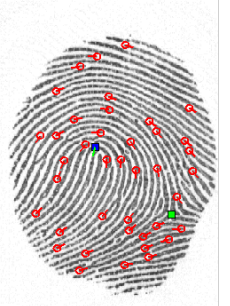

(a)

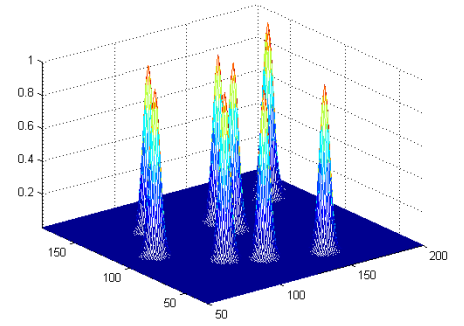

(b)

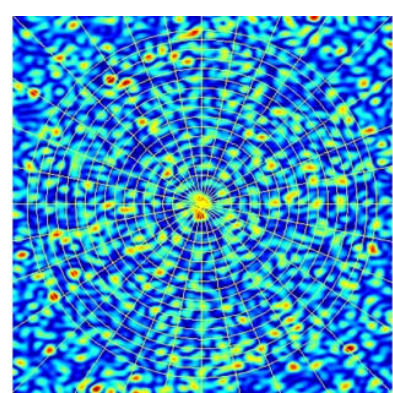

(c)

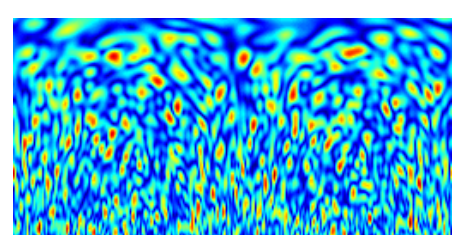

(d)

Figure 3. Illustration of the complex spectral minutiae representation procedure. (a) a fingerprint and its minutiae; (b) representation of minutiae points as complex valued continuous functions; (c) the 2D Fourier spectrum of ' $b$ ' in a Cartesian coordinate and a polar sampling grid; (d) the Fourier spectrum sampled on a polar grid.

scheme based on fuzzy commitment and helper data scheme, illustrated in Figure 1. In Figure 2, we show the various processing modules of the spectral minutiae representation scheme. The main modules of this scheme are: Complex Spectral Minutiae representation (SMC), Feature Reduction (FR), Quantization (Q), Fractional Hamming Distance (FHD). In this section, we will introduce each module respectively.

\section{B. Complex Spectral Minutiae Representation}

The objective of the spectral minutiae representation is to represent a minutiae set as a fixed-length feature vector, which is invariant to translation and rotation [2]. We assume that the scaling has already been compensated for on the level of the minutiae sets. This is for instance possible if minutiae are presented in a standard like [6], which includes sensor resolution. In Figure 3, the procedure of the complex spectral minutiae representation (SMC) is illustrated.

Assume a fingerprint with $Z$ minutiae. First, we code the minutiae locations by indicator functions, which are isotropic two-dimensional Gaussian kernels in the spatial domain. Then we incorporate the minutiae orientation by assigning each Gaussian a complex amplitude $\mathrm{e}^{\mathrm{j} \theta_{i}}, i=$ $1, \ldots, Z$. In this way, we represent minutiae points as complex valued continuous functions, the magnitude of which is shown in Figure 3(b). In this representation, translation and rotation may exist, depending on how the user has put his finger on the sensor.

Next, a two-dimensional continuous Fourier transform is performed and only the Fourier magnitude is kept, illustrated in Figure 3(c). This representation is now translation invariant according to the shift property of the continuous Fourier transform. In addition, we incorporate the minutiae quality data as presented in [7] into SMC. This representation can be computed analytically,

$$
\begin{aligned}
& \mathcal{M}_{\mathrm{C}}\left(\omega_{\mathrm{x}}, \omega_{\mathrm{y}} ; \sigma_{\mathrm{C}}^{2}\right)= \\
& \left|\exp \left(-\frac{\omega_{\mathrm{x}}^{2}+\omega_{\mathrm{y}}^{2}}{2 \sigma_{\mathrm{C}}^{-2}}\right) \sum_{i=1}^{Z} w_{i} \exp \left(-\mathrm{j}\left(\omega_{\mathrm{x}} x_{i}+\omega_{\mathrm{y}} y_{i}\right)+\mathrm{j} \theta_{i}\right)\right|,
\end{aligned}
$$

with $\left(x_{i}, y_{i}, \theta_{i}, w_{i}\right)$ the location, orientation and quality of the $i$-th minutia in the fingerprint, and $\left(\omega_{\mathrm{x}}, \omega_{\mathrm{y}} ; \sigma_{\mathrm{C}}^{2}\right)$ are the frequencies and the parameters of the Gaussian kernel function respectively.

Finally, the Fourier spectrum is re-mapped onto a polar coordinate system, illustrated in Figure 3(d). In the radial direction $\lambda$, we use $M=128$ samples between $\lambda_{1}=0.05$ and $\lambda_{\mathrm{h}}=0.58$. In the angular direction $\beta$, we use $N=256$ samples uniformly distributed between $\beta=0$ and $\beta=2 \pi$. Since our target application is in a high security scenario with reasonable good quality fingerprints, we choose $\sigma_{\mathrm{C}}=$ 0 for the best good performance. In this case, there is no multiplication with a Gaussian in the frequency domain (an analysis of the selection of the Gaussian parameter $\sigma$ can be found in [2]). According to the rotation properties of the two-dimensional continuous Fourier transform, now the rotation becomes translation along the new coordinate axis.

Let $R(m, n)$ and $T(m, n)$ be the two sampled minutiae spectra, respectively, achieved from the reference fingerprint and test fingerprint. Both $R(m, n)$ and $T(m, n)$ are normalized to have zero mean and unit energy. We can use the two-dimensional correlation coefficient between $R$ and $T$ as a measure of their similarity.

In practice, the input fingerprint images are rotated. Therefore, we need to test a few rotations, which become the circular shifts in the horizontal direction. We denote $T(m, n-j)$ as a circularly shifted version of $T(m, n)$, the final matching score between $R$ and $T$ is, 


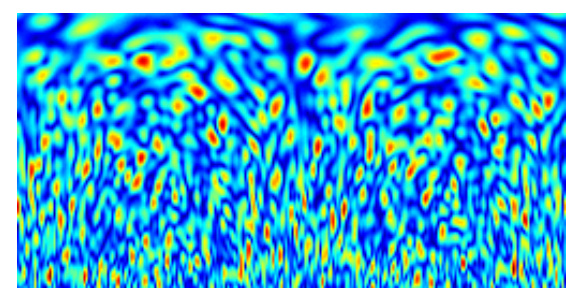

(a)

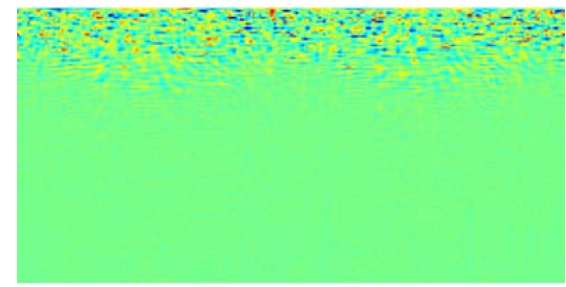

(b)

Figure 4. Illustration of the CPCA transform. (a) the SMC feature; (b) the minutiae spectrum after the CPCA transform.

$$
\begin{array}{r}
S^{(R, T)}=\max _{j}\left\{\frac{1}{M N} \sum_{m, n} R(m, n) T(m, n-j)\right\}, \\
-15 \leq j \leq 15 .
\end{array}
$$

\section{Feature Reduction}

The spectral minutiae feature is a 32,768-dimensional real-valued feature vector. This large dimensionality of the spectral minutiae feature can cause three problems. First, the template storage requirement is very high. Second, the high dimensionality leads to a computational burden and the matching speed will be limited. Third, the high dimensionality can lead to a small sample size problem [8]. In order to cope with these problems, we will apply the Column Principal Component Analysis (CPCA) feature reduction method introduced in [9].

The idea of CPCA is to apply the well known Principal Component Analysis (PCA) technique to the SMC columns. PCA has two functions: it decorrelates features and concentrates power. The CPCA representation is shown in Figure 4(b). We can see that after CPCA, the power is concentrated in the upper lines. The features in the lower parts are close to zero, so we can remove them from the representation. For the CPCA feature reduction, we keep the top 40 lines, with a feature reduction rate of $69 \%$.

\section{Quantization}

A fixed-length binary representation of a fingerprint has the advantages of a fast operation and a small template storage. For many biometric template protection schemes, a binary string is also required as input. The Spectral Bits quantization is applied to the real-valued features after the CPCA feature reduction. First, each real-valued feature is

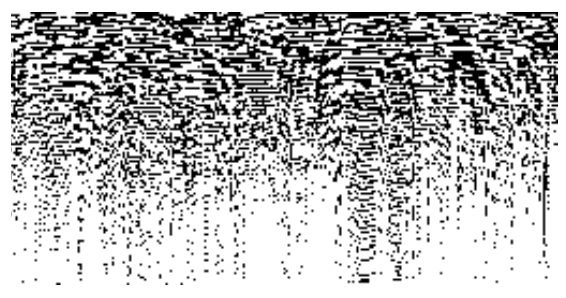

(a)

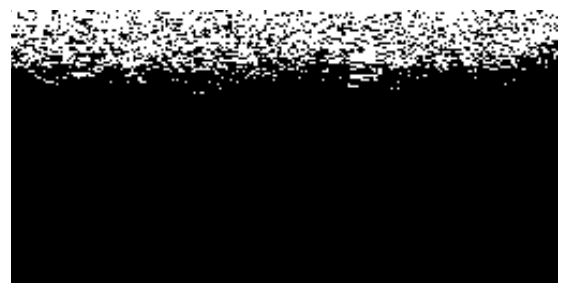

(b)

Figure 5. Example of Spectral Bits (SMC spectra after CPCA). (a) the Sign Bit; (b) the Mask Bit.

quantized as one bit ( 1 if the feature is greater than zero and 0 otherwise, we call it sign bit), shown in Figure 5(a). Second, since the quantization boundary is zero, and the features close to zero are unstable and likely to flip, they may cause errors. Therefore, we will mask out the features of which the absolute values are below a certain threshold. For the best recognition performance, we set the threshold to 0.6 after normalizing the spectra to have a standard deviation (STD) equal to 1. By testing different thresholds on different fingerprint databases, we found out that this parameter can be chosen empirically and it will not cause critical degradation of recognition performance. An example of the resulting mask bit is shown in Figure 5(b).

\section{E. Fractional Hamming Distance}

After generating sign bit and mask bit vectors, we can compute a Fractional Hamming distance (FHD) [10] as a measure of the dissimilarity between two fingerprints spectra $R(m, n)$ and $T(m, n)$, whose sign bit vectors are denoted $\{$ codeR, codeT $\}$ and whose mask bit vectors are decoded $\{$ maskR, maskT $\}$,

$$
F H D=\frac{\|(\operatorname{codeR} \otimes \operatorname{code} \mathrm{T}) \cap \operatorname{maskR} \cap \operatorname{maskT}\|}{\|\operatorname{maskR} \cap \operatorname{maskT}\|} .
$$

\section{Multi-SAMPlE FUSION OF THE SPECTRAL MINUTIAE REPRESENTATIONS}

Biometric fusion is a commonly used approach to improve the recognition performance of a biometric system by combining multiple sources of biometric information. In this paper, we will investigate the strategies of fusing multiple fingerprint samples (obtained from the same sensor) at the feature- and decision-level respectively. 


\section{A. Feature-level fusion}

During the Feature-level fusion, the features from multiple samples are combined to produce a single enrollment template. This is also known as template consolidation [1]. Regarding the spectral minutiae representation scheme shown in Figure 2, the feature-level fusion can be performed at the modules 'Complex Spectral Minutiae representation (SMC)', 'Feature Reduction (FR)' and 'Quantization (Q)'. At each module, the amount of information available is different (the information available decreases from left to right in the figure). Considering the information available, in this paper, we will perform the spectral minutiae features fusion after the CPCA feature reduction (module FR).

In the spectral minutiae representations, the translations between fingerprint samples become invariant, while the rotations become the circular shifts in the horizontal direction. Before the feature-level fusion, we need to first align the spectral minutiae features to compensate the rotation differences. After the rotation alignment, we average the aligned spectral minutiae features to generate the synthesized enrollment (or reference) template.

Assume we have $N_{\mathrm{E}}$ enrollment spectral minutiae representation samples $R_{1}, \ldots, R_{N_{\mathrm{E}}}$ available for fusion, the procedure of our spectral minutiae feature-level fusion is as follows.

Step 1: Denote $R_{i^{*}}$ as the enrollment sample with the largest similarity to all the other samples, that is,

$$
i^{*}=\arg \max _{i} \sum_{\substack{k=1 \\ k \neq i)}}^{N_{\mathrm{E}}} S^{\left(R_{i}, R_{k}\right)}, i=1, \ldots, N_{\mathrm{E}},
$$

with $S^{\left(R_{i}, R_{k}\right)}$ calculated following Equation (2).

Step 2: Take $R_{i^{*}}$ as the reference, align all the other enrollment samples to $R_{i^{*}}$ by trying out different circular shifts following Equation (2). The aligned samples are denoted as $\widetilde{R}_{1}, \ldots, \widetilde{R}_{N_{\mathrm{E}}}$.

Step 3: Generate the the synthesized enrollment template $R_{\mathrm{S}}$ by averaging $\widetilde{R}_{1}, \ldots, \widetilde{R}_{N_{\mathrm{E}}}$, that is,

$$
R_{\mathrm{S}}=\frac{1}{N_{\mathrm{E}}} \sum_{i=1}^{N_{\mathrm{E}}} \widetilde{R}_{i} .
$$

Finally, the synthesized enrollment template $R_{\mathrm{S}}$ will be stored in the database as the reference template for verification/identification.

\section{B. Decision-level fusion}

The decision-level fusion is performed at the final decision making module, see Figure 2. The very straightforward decision-level fusion techniques are AND Rule, OR Rule and Majority Voting [11]. The outliers in a fingerprint database can cause false rejection. To reduce the recognition errors caused by the outliers, in this paper, we show the
Table I

PERMUTATION SETTING: SAMPLES USED FOR MULTI-SAMPLE ENROLLMENT AND SINGLE-SAMPLE VERIFICATION.

\begin{tabular}{|c|c|c|}
\hline Permutation & Enrollment & Genuine Verification \\
\hline \hline P1 & $1,2,3,4$ & $5,6,7,8$ \\
\hline P2 & $1,3,5,7$ & $2,4,6,8$ \\
\hline P3 & $1,2,7,8$ & $3,4,5,6$ \\
\hline P4 & $1,5,6,7$ & $2,3,4,8$ \\
\hline
\end{tabular}

performance of the decision-level fusion based on the OR Rule in Section IV ${ }^{1}$.

\section{RESUlts}

The proposed algorithms have been evaluated on the FVC2000-DB2 [12] fingerprint database. We apply the same experimental protocol as in the FVC competition: the samples from finger ID 101 to 110 for the CPCA training and samples from person ID 1 to 100 for test. Each identity contributes 8 samples. The minutiae sets including the minutiae quality data are extracted by a proprietary method.

We test our algorithm in a verification setting. In the single-sample enrollment case, for genuine comparisons, we used all the possible combinations. For imposter comparisons, we chose the first sample from each identity. Therefore, we generate $100 \times\left(\begin{array}{l}8 \\ 2\end{array}\right)=2800$ genuine comparisons and $\left(\begin{array}{c}100 \\ 2\end{array}\right)=4950$ imposter comparisons in total.

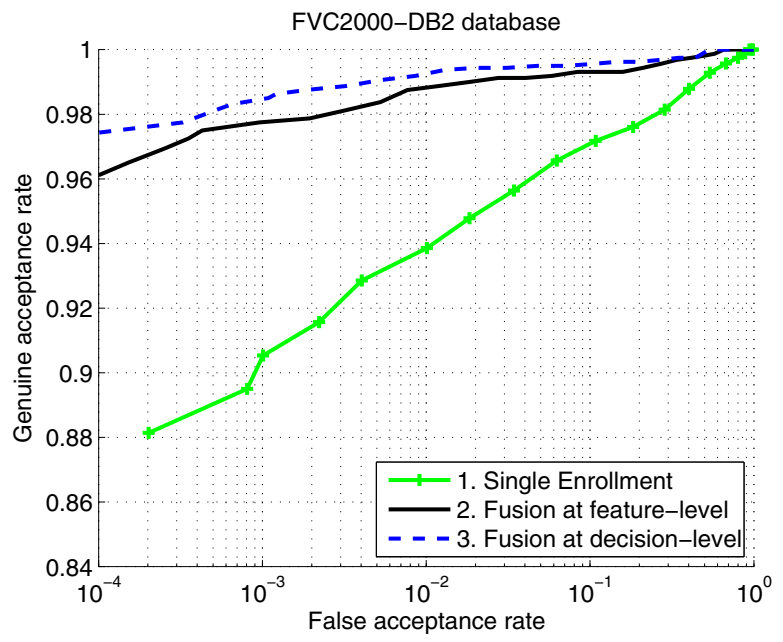

Figure 6. ROC curves of the proposed schemes.

To test different multi-sample fusion schemes proposed in Section III, we set up a multi-sample enrollment and single-sample verification system. We use the Fractional Hamming Distance shown in Equation (3) as the classifier. For generating more test cases, we implemented four permutations. In each permutation, $N_{\mathrm{E}}=4$ enrollment

\footnotetext{
${ }^{1}$ We also tried AND Rule and Majority Voting. The OR Rule fusion gives best results in our case.
} 
samples are used for multi-sample fusions and the other four samples for genuine verification. For imposter verification, we chose the first sample from each identity to compare with the multiple enrollment samples (or the synthesized enrollment template in the feature-level fusion case). The permutation setting is shown in Table I. In total, we will generate $100 \times 4 \times 4=1600$ genuine comparisons and $100 \times 99 \times 4=39600$ imposter comparisons.

For comparison, we present the results of the singleenrollment scheme and two multi-sample fusion schemes. The ROC curves of each scheme are shown in Figure 6. For the multi-sample fusion results, we can see that both feature- and decision-level fusion schemes received significant improvements compared with the single-enrollment scheme. The OR Rule decision-level fusion outperformed the feature-level fusion since it is more robust to outliers and the overfitting problem.

\section{CONCLuSion}

Minutiae-based matching is the most widely used technique in fingerprint recognition systems. However, the low matching speed is limiting their application. At the same time, the increasing security and privacy concerns make minutiae template protection one of the most crucial tasks. The spectral minutiae representation method has coped with the above disadvantages.

In this paper, we present the spectral minutiae representation scheme. We also introduced multi-sample fusion schemes to enhance the recognition performance. The experiments showed promising results.

To apply the spectral minutiae representation with a template protection scheme based on the helper data scheme [5], an error correction scheme is needed. Furthermore, to enhance the recognition performance, we can incorporate other fingerprint features such as singular points. Investigating the possible error correction codes and other methods to enhance recognition performance will be our future work.

\section{ACKNOWLEDGMENT}

This research is supported by the ProBiTe project funding by Sentinels and the TURBINE project funding by the European Union under the Seventh Framework Programme.

\section{REFERENCES}

[1] D. Maltoni, D. Maio, A. K. Jain, and S. Prabhakar, Handbook of Fingerprint Recognition. Springer Publishing Company, Incorporated, 2009.

[2] H. Xu, R. Veldhuis, A. Bazen, T. Kevenaar, T. Akkermans, and B. Gokberk, "Fingerprint verification using spectral minutiae representations," Information Forensics and Security, IEEE Transactions on, vol. 4, no. 3, pp. 397-409, Sept. 2009.

[3] H. Xu and R. Veldhuis, "Complex spectral minutiae representation for fingerprint recognition," in Proceedings of the IEEE Computer Society Conference on Computer Vision and Pattern Recognition - Workshop on Biometrics, San Francisco, USA, June 2010.

[4] A. Juels, "Fuzzy commitment," in Security with Noisy Data - On Private Biometrics, Secure Key Storage and AntiCounterfeiting, P. Tuyls, B. Skoric, and T. Kevenaar, Eds. Springer, 2007, ch. 3, pp. 45-56.

[5] P. Tuyls, A. Akkermans, T. Kevenaar, G. Schrijen, A. Bazen, and R. Veldhuis, "Practical biometric authentication with template protection." in AVBPA, 2005, pp. 436-446.

[6] "ISO/IEC 19794-2, Information Technology - Biometric Data Interchange Format - Part 2: Finger Minutiae Data," 2005.

[7] H. Xu and R. Veldhuis, "Spectral minutiae representatoins of fingerprints enhanced by quality data," in IEEE Third International Conference on Biometrics: Theory, Applications and Systems (BTAS '09), September 2009.

[8] S. Raudys and A. Jain, "Small sample size effects in statistical pattern recognition: Recommendations for practitioners," vol. 13, no. 3, pp. 252-264, March 1991.

[9] H. Xu, R. Veldhuis, T. Kevenaar, and T. Akkermans, "A fast minutiae-based fingerprint recognition system," Systems Journal, IEEE, vol. 3, no. 4, pp. 418 -427, dec. 2009.

[10] J. Daugman, "The importance of being random: Statistical principles of iris recognition," Pattern Recognition, vol. 36, no. 2, pp. 279-291, 2003.

[11] A. A. Ross, K. Nandakumar, and A. K. Jain, Handbook of Multibiometrics (International Series on Biometrics). Secaucus, NJ, USA: Springer-Verlag New York, Inc., 2006.

[12] D. Maio, D. Maltoni, R. Cappelli, J. Wayman, and A. Jain, "FVC2000: Fingerprint verification competition," IEEE Trans. PAMI, vol. 24, no. 3, pp. 402-412, Mar. 2002. 